\title{
Design of Adjustable Square-Shaped 2D IIR Filters
}

\author{
Radu Matei \\ Faculty of Electronics and Telecommunications, "Gh. Asachi” Technical University, Boulevard Carol I No. 11, 700506 Iaşi, Romania
}

Correspondence should be addressed to Radu Matei; rmatei@etti.tuiasi.ro

Received 28 June 2013; Accepted 28 July 2013

Academic Editors: V. Chandran and W. Zuo

Copyright (c) 2013 Radu Matei. This is an open access article distributed under the Creative Commons Attribution License, which permits unrestricted use, distribution, and reproduction in any medium, provided the original work is properly cited.

\begin{abstract}
This paper proposes an analytical design method for two-dimensional square-shaped IIR filters. The designed 2D filters are adjustable since their bandwidth and orientation are specified by parameters appearing explicitly in the filter matrices. The design relies on a zero-phase low-pass $1 \mathrm{D}$ prototype filter. To this filter a frequency transformation is next applied, which yields a 2D filter with the desired square shape in the frequency plane. The proposed method combines the analytical approach with numerical approximations. Since the prototype transfer function is factorized into partial functions, the 2D filter also will be described by a factorized transfer function, which is an advantage in implementation.
\end{abstract}

\section{Introduction}

Various design methods for 2D filters, both FIR and IIR, have been proposed by many researchers [1]. A frequently used design technique is based on a specified $1 \mathrm{D}$ prototype filter, whose transfer function is transformed using various frequency mappings, in order to obtain a 2D filter with a desired frequency response. Some relevant papers approaching $2 \mathrm{D}$ filter design using spectral transformations are [2-6]. A class of tunable 2D digital filters is discussed in [7]. The stability problem for $2 \mathrm{D}$ filters and stabilization methods are treated in papers like [8-11].

Diamond filters have been used as antialiasing filters in the conversion between signals sampled on the rectangular sampling grid and the quincunx sampling grid. Different issues related to design methods for diamond filters were studied in [12-14]. In [15], another design method for diamond-shaped filters was derived, starting from discrete filter transfer functions; two types of filters were obtained, one with complex transfer function and another with zerophase transfer function. The latter is particularly appropriate for image processing applications as the filters introduce no phase distortions. The technique developed in [15] uses the 2D filter specification in polar coordinates.

In this paper an analytical design method is proposed for 2D adjustable zero-phase square-shaped filters, a larger class of filters which may be regarded as a generalization of the common diamond filter. Starting from a 1D prototype filter with factorized transfer function, the corresponding $2 \mathrm{D}$ filters are obtained by a particular 1D to 2D frequency mapping. The 2D filter will have a factorized transfer function, which is a useful feature in implementation. This work mainly focuses on presenting the proposed method and describes in detail the design steps. Several design examples are also provided. The typical applications of diamond filters were not approached here, as they are extensively treated in many other works. Image processing applications of the square-shaped filters with arbitrary bandwidth and orientation proposed here will be approached in further work.

\section{Specification of Square-Shaped Filters}

2.1. Frequency Plane Specification of Square-Shaped Filters. A particular case of a square-shaped filter is the standard diamond filter; its shape in the frequency plane is shown in Figure 1(a). It is a square with a side length of $\pi \sqrt{2}$, while its axis is tilted by an angle of $\varphi=\pi / 4$ radians about the two frequency axes. Next we will consider the orientation angle $\varphi$ about the $\omega_{2}$ - axis.

In this work a more general case is approached, that is, a 2D diamond-type filter with a square shape in the frequency plane but with arbitrary side length and axis inclination angle, as shown in Figure 1(e). Next we refer to them as 


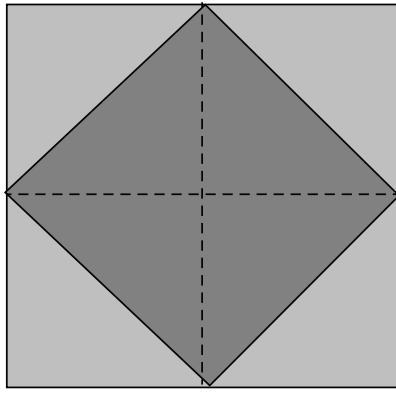

(a)

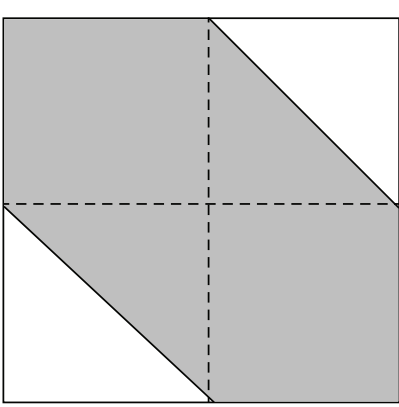

(b)

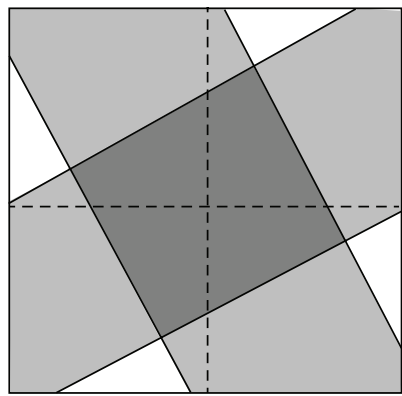

(e)

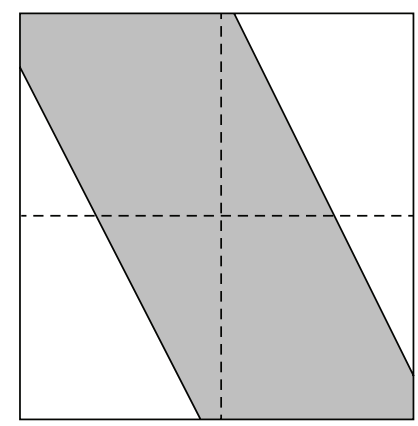

(c)

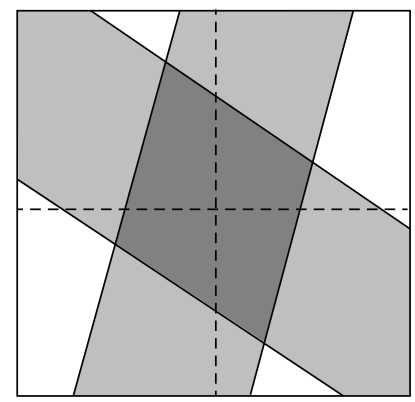

(f)

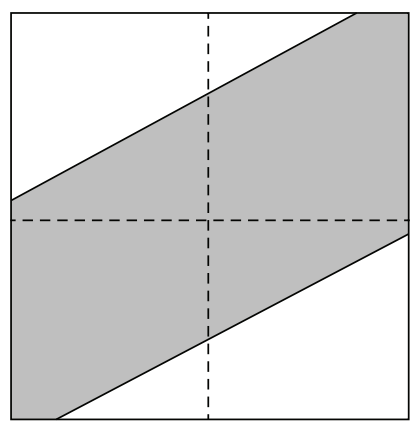

(d)

FIGURE 1: (a) diamond filter; (b) wide-band oriented filter; (c), (d) wide-band oriented filters with orientations forming an angle $\varphi=\pi / 2$; (e) square-shaped filter resulted as product of the above oriented filters; (f) rhomboidal filter.

square-shaped filters, since they are more general than the diamond filter from Figure 1(a).

The square-shaped filter in Figure 1(e) is derived as the intersection of two oriented low-pass filters whose axes are perpendicular to each other, for which the shape in the frequency plane is given in Figures 1(c) and 1(d). Correspondingly, the square-shaped filter transfer function $H_{S}\left(z_{1}, z_{2}\right)$ results as a product of two partial transfer functions $H_{D 1}\left(z_{1}, z_{2}\right)$ and $H_{D 2}\left(z_{1}, z_{2}\right)$, which we refer to as directional filters:

$$
H_{S}\left(z_{1}, z_{2}\right)=H_{D 1}\left(z_{1}, z_{2}\right) \cdot H_{D 2}\left(z_{1}, z_{2}\right)
$$

The frequency response of $H_{D 2}\left(z_{1}, z_{2}\right)$ is ideally identical to the frequency response of $H_{D 1}\left(z_{1}, z_{2}\right)$, rotated by an angle of $\varphi=\pi / 2$. Since this axis rotation implies the frequency variable change, $\omega_{1} \rightarrow \omega_{2}, \omega_{2} \rightarrow-\omega_{1}$, the transfer function $H_{D 2}\left(z_{1}, z_{2}\right)$ can be derived from $H_{D 1}\left(z_{1}, z_{2}\right)$ as

$$
H_{D 2}\left(z_{1}, z_{2}\right)=H_{D 1}\left(z_{2}, z_{1}^{-1}\right) .
$$

A more general filter belonging to this class is a rhomboidal filter, as shown in Figure 1(f). In this case the two oriented low-pass component filters may have different bandwidths and their axes are no longer perpendicular to each other.
2.2.1D Low-Pass Zero-Phase Prototype Filter. An analog filter of order $N$ is described by the general transfer function in variable $s$ :

$$
H_{P}(s)=\frac{P(s)}{Q(s)}=\frac{\sum_{i=0}^{M} p_{i} \cdot s^{i}}{\sum_{j=0}^{N} q_{j} \cdot s^{j}} .
$$

A zero-phase prototype can be obtained from the general filter $H_{P}(s)$ if the magnitude characteristics $\left|H_{P}(j \omega)\right|$ are considered. Zero-phase filters are frequently used in image processing as they do not introduce any phase distortions in the filtered image. In order to obtain a maximally flat or lowripple square-shaped filter, we start from a maximally flat $1 \mathrm{D}$ prototype.

Let us consider a Butterworth low-pass filter $H_{B}(s)$ of order $N$, having the transfer function magnitude as follows:

$$
\left|H_{B}(j \omega)\right|=\frac{1}{\sqrt{1+\left(\omega / \omega_{0}\right)^{2 N}}},
$$

where $\omega_{0}$ is the filter cut-off frequency.

We look for a rational expression of the magnitude $\left|H_{B}(j \omega)\right|$, which has to be an approximation as accurate as possible within a specified error on the frequency domain $[-\pi, \pi]$. The most convenient for our purpose is the Chebyshev-Padé expansion, since it yields an efficient approximation of a given function, which is uniform along the entire specified interval. It can be found using a symbolic computation software like MAPLE. 


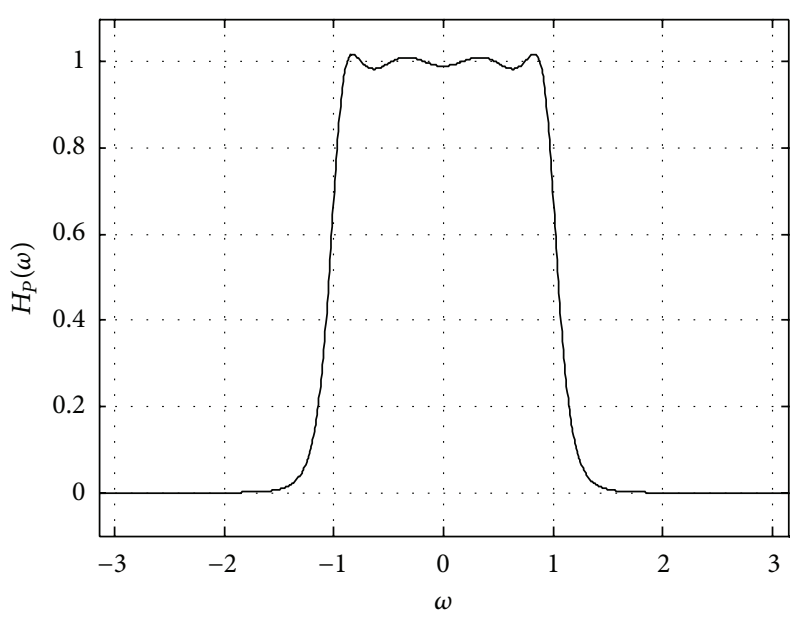

(a)

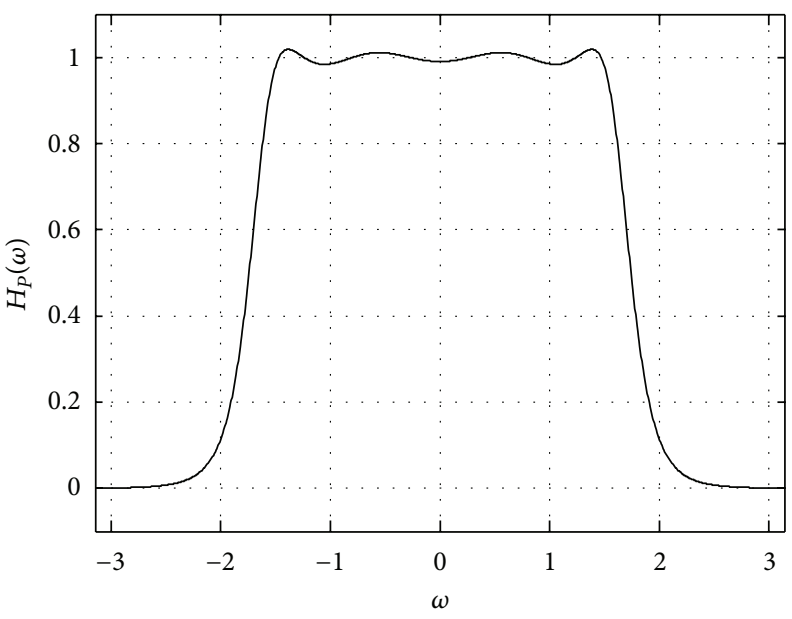

(b)

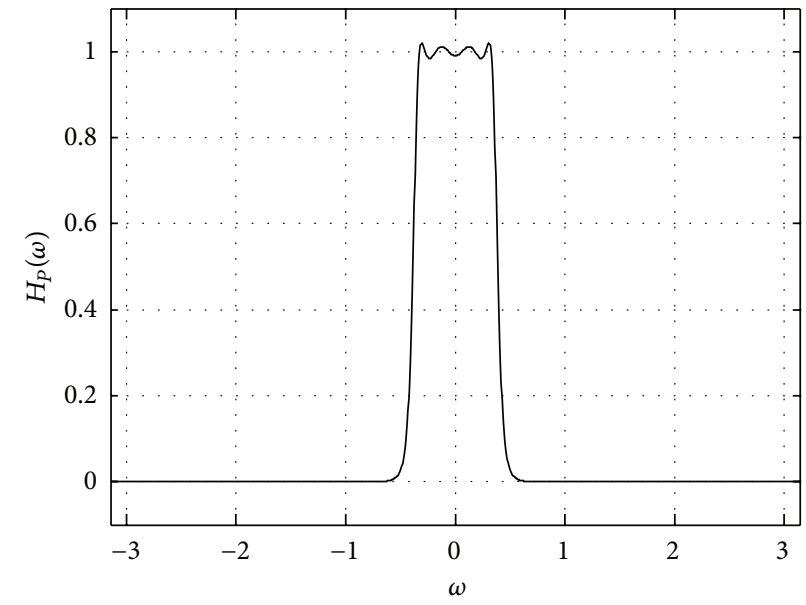

(c)

FIGURE 2: (a) Zero-phase low-pass prototype filter characteristic for $p=1\left(\omega_{0}=1\right)$; (b), (c) scaled LP characteristics for $p=0.6\left(\omega_{0}=0.53 \pi\right)$ and $p=2.7\left(\omega_{0}=0.118 \pi\right)$.

Let us first find the approximation for the function (4) in normalized frequency, that is, the function $\left|H_{B n}(j \omega)\right|$ :

$$
\left|H_{B n}(j \omega)\right|=\frac{1}{\sqrt{1+\omega^{2 N}}}
$$

Since a steeper filter would be desirable, let us take a Butterworth low-pass filter of order $N=12$; we obtain on the frequency range $[-\pi, \pi]$ the following ChebyshevPadé rational approximation of order 8 for the magnitude characteristics, as a ratio of polynomials with the same degree 8 , which in factorized form looks like

$$
\begin{aligned}
& \frac{1}{\sqrt{1+\omega^{24}}} \\
& \quad \cong H_{P}(\omega)=\xi \cdot \frac{\left(\omega^{4}-5.8589322 \cdot \omega^{2}+22.393616\right)}{\left(\omega^{4}+0.253253 \cdot \omega^{2}+0.2830378\right)}
\end{aligned}
$$

$$
\begin{aligned}
& \cdot \frac{\left(\omega^{4}-13.689786 \cdot \omega^{2}+49.701196\right)}{\left(\omega^{4}-1.843889 \cdot \omega^{2}+0.999034\right)} \\
& =\xi \cdot H_{P 1}(\omega) \cdot H_{P 2}(\omega),
\end{aligned}
$$

where the constant has the value $\xi=0.0002516$.

The frequency response $H_{P}(\omega)$ of this prototype is plotted in Figure 2(a) and shows a small amplitude ripple in the pass-band. The cut-off frequency is $\omega_{0}=1$ and $p=1$. A low-pass filter with a better flatness would obviously have a higher order. The advantage of using this factorized rational approximation is that it is easily scalable on the frequency axis, as will appear clearly in the following sections.

Each of the rational expressions $H_{P i}(\omega)(i=1,2, \ldots)$ occurring as factors in $H_{P}(\omega)$ given by (6), with 4 th order polynomials as numerator and denominator, can be generally written as

$$
H_{P i}(\omega)=\frac{\left(\omega^{4}+b_{1 i} \cdot \omega^{2}+b_{0 i}\right)}{\left(\omega^{4}+a_{1 i} \cdot \omega^{2}+a_{0 i}\right)} \quad(i=1,2, \ldots) .
$$


Of course, in a factorized rational approximation like expression (6) there may occur also a second-order term:

$$
H_{P i}(\omega)=\frac{\left(\omega^{2}+c_{0 i}\right)}{\left(\omega^{2}+d_{0 i}\right)}
$$

but this case is not further considered.

As specified earlier, the variable $\omega$ represents here the frequency normalized to the value of the cut-off frequency $\omega_{0}$. In order to return to current frequency values, in the expression of the general factor $H_{P i}(\omega)$, we must substitute $\omega$ by $\omega / \omega_{0}$. It is more convenient to substitute $\omega$ by $p \cdot \omega$, where $p=1 / \omega_{0}$. Thus the ratio factor $H_{P i}(\omega)$ from (7) can be rewritten in the following form which includes the parameter $p$, which makes it scalable along the frequency axis:

$$
H_{P i}(\omega)=\frac{p^{4} \omega^{4}+b_{1 i} \cdot p^{2} \omega^{2}+b_{0 i}}{p^{4} \omega^{4}+a_{1 i} \cdot p^{2} \omega^{2}+a_{0 i}} .
$$

For a value of the parameter $p$ different from unity, the characteristic $H_{P}(\omega)$ displayed in Figure 2 either stretches (for $p<1$ ) or shrinks (for $p>1$ ). In Figures 2(b) and 2(c) two LP characteristics derived from $H_{P}(\omega)$ are plotted, for the indicated values of $p$ and $\omega_{0}$, respectively.

Thus the $1 \mathrm{D}$ prototype is parametric depending on $p$, and the derived $2 \mathrm{D}$ square-shaped filter will have an adjustable pass-band region, specified by the same $p$.

\section{Design Method}

The main issue in this section is to find the transfer function of the desired 2D square filter $H_{S}\left(z_{1}, z_{2}\right)$ using a particular frequency transformation.

From a $1 \mathrm{D}$ prototype filter $H_{P}(s)=H_{P}(j \omega)$ (which varies on one axis only), a $2 \mathrm{D}$ oriented filter may be obtained by rotating the axes of the plane $\left(\omega_{1}, \omega_{2}\right)$ by an angle $\varphi$. The rotation is defined by the following linear transformation, where $\omega_{1}, \omega_{2}$ are the original frequency variables and $\bar{\omega}_{1}, \bar{\omega}_{2}$ the rotated ones [1]:

$$
\left[\begin{array}{l}
\omega_{1} \\
\omega_{2}
\end{array}\right]=\left[\begin{array}{cc}
\cos \varphi & \sin \varphi \\
-\sin \varphi & \cos \varphi
\end{array}\right] \cdot\left[\begin{array}{l}
\bar{\omega}_{1} \\
\bar{\omega}_{2}
\end{array}\right] .
$$

The spatial orientation is specified by an angle $\varphi$ with respect to $\omega_{1}-$ axis, defined by the following $1 \mathrm{D}$ to $2 \mathrm{D}$ frequency mapping:

$$
\omega \longrightarrow \omega_{1} \cos \varphi+\omega_{2} \sin \varphi
$$

The oriented filter transfer function results in the following:

$$
H_{\varphi}\left(\omega_{1}, \omega_{2}\right)=H_{P}\left(\omega_{1} \cos \varphi+\omega_{2} \sin \varphi\right) .
$$

In the complex plane $\left(s_{1}, s_{2}\right)$ the frequency transformation (11) becomes

$$
s \longrightarrow s_{1} \cos \varphi+s_{2} \sin \varphi .
$$

The $2 \mathrm{D}$ oriented filter $H_{\varphi}\left(\omega_{1}, \omega_{2}\right)$ has the magnitude section along the line $\omega_{1} \cos \varphi+\omega_{2} \sin \varphi=0$, identical with the prototype $H_{P}(\omega)$, and is constant along the perpendicular line: $\omega_{1} \sin \varphi-\omega_{2} \cos \varphi=0$ (the filter longitudinal axis).

In our case, since the rational prototype function $H_{P}(\omega)$ from (6) contains only even powers of frequency $\omega$, that is, only powers of $\omega^{2}$, we will derive a convenient expression for $\Omega_{\varphi}=\left(\omega_{1} \cos \varphi+\omega_{2} \sin \varphi\right)^{2}$, according to mapping (11):

$$
\begin{aligned}
\Omega_{\varphi}= & 0.5 \sin 2 \varphi \cdot\left(\omega_{1}+\omega_{2}\right)^{2} \\
& +0.5(1+\cos 2 \varphi-\sin 2 \varphi) \cdot \omega_{1}^{2} \\
& +0.5(1-\cos 2 \varphi-\sin 2 \varphi) \cdot \omega_{2}^{2} .
\end{aligned}
$$

Thus using (11) we derived the mapping $\omega^{2} \rightarrow \Omega_{\varphi}$. Since $s_{1}^{2}=-\omega_{1}^{2}, s_{2}^{2}=-\omega_{2}^{2}$, the corresponding expression $S_{\varphi}$ in the complex variables $s_{1}$ and $s_{2}$ is similar, with $S_{\varphi}=-\Omega_{\varphi}$ :

$$
\begin{aligned}
S_{\varphi}= & \left(s_{1} \cos \varphi+s_{2} \sin \varphi\right)^{2} \\
= & 0.5 \sin 2 \varphi \cdot\left(s_{1}+s_{2}\right)^{2} \\
& +0.5(1+\cos 2 \varphi-\sin 2 \varphi) \cdot s_{1}^{2} \\
& +0.5(1-\cos 2 \varphi-\sin 2 \varphi) \cdot s_{2}^{2} .
\end{aligned}
$$

Therefore, after applying the $1 \mathrm{D}$ to $2 \mathrm{D}$ directional mapping (11) by substituting $\omega^{2} \rightarrow \Omega_{\varphi}=-S_{\varphi}$, we reach the following rational expression in the complex frequency variables $s_{1}, s_{2}$, which corresponds to each ratio factor $H_{P i}(\omega)$ of the prototype frequency response, given by (9)

$$
F_{D i}\left(s_{1}, s_{2}\right)=\frac{p^{4} S_{\varphi}^{2}-b_{1 i} \cdot p^{2} S_{\varphi}+b_{0 i}}{p^{4} S_{\varphi}^{2}-a_{1 i} \cdot p^{2} S_{\varphi}+a_{0 i}} .
$$

Since finally we must reach a transfer function of a discrete $2 \mathrm{D}$ filter in the complex variables $z_{1}$ and $z_{2}$, we must determine the discrete counterpart $H_{D i}\left(z_{1}, z_{2}\right)$ of the general factor $F_{D i}\left(s_{1}, s_{2}\right)$.

The currently used method to obtain a discrete filter from an analog prototype is applying the bilinear transform. If the sample interval takes the value $T=1$, the bilinear transform for $s_{1}$ and $s_{2}$ in the complex plane $\left(s_{1}, s_{2}\right)$ has the form

$$
\begin{aligned}
& s_{1}=\frac{2\left(z_{1}-1\right)}{\left(z_{1}+1\right)}, \\
& s_{2}=\frac{2\left(z_{2}-1\right)}{\left(z_{2}+1\right)} .
\end{aligned}
$$

Even if this method is straightforward, the designed $2 \mathrm{D}$ filter, corresponding to the transfer function in $z_{1}, z_{2}$, will inherently present substantial linearity distortions towards the limits of the frequency plane as compared to the ideal frequency response. This fact is mainly due to the so-called frequency warping effect of the bilinear transform, expressed by the continuous to discrete frequency mapping:

$$
\omega=\frac{2}{T} \cdot \operatorname{arctg}\left(\frac{\omega_{a} T}{2}\right)
$$




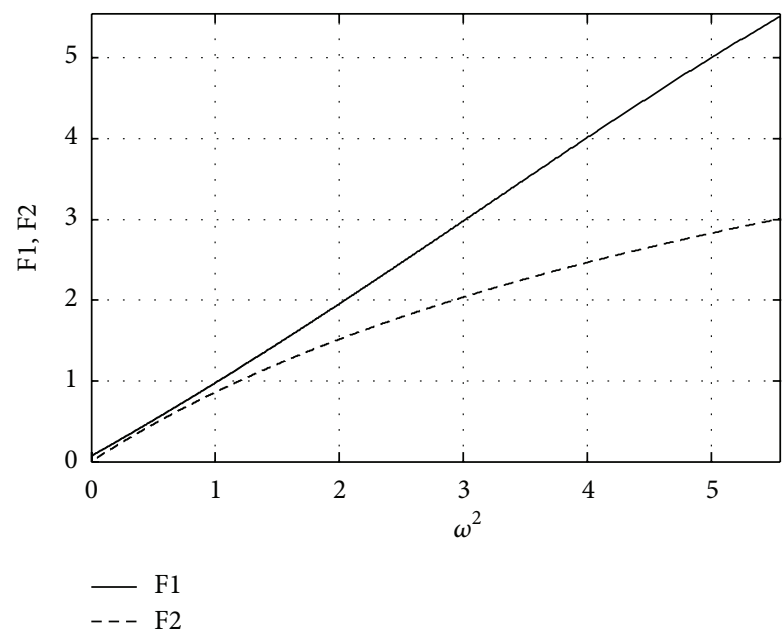

FIgURE 3: Plots of mapping (26) - F1 in solid line, and mapping (21) - F2 in dash line.

where $\omega$ is a frequency of the discrete filter and $\omega_{a}$ is the corresponding frequency of the analog filter. This error can be corrected by applying a prewarping. Taking $T=1$ in relation (18) we obtain the mappings

$$
\begin{aligned}
& \omega_{1} \longrightarrow 2 \cdot \operatorname{arctg}\left(\frac{\omega_{1}}{2}\right), \\
& \omega_{2} \longrightarrow 2 \cdot \operatorname{arctg}\left(\frac{\omega_{2}}{2}\right) .
\end{aligned}
$$

In order to include the nonlinear mappings (19) into the frequency transformation, a rational approximation would be needed. Using again the efficient Chebyshev-Padé method, we get the following accurate approximation on the range $[-\pi, \pi]$ :

$$
\operatorname{arctg}\left(\frac{\omega}{2}\right) \cong 0.4751 \cdot \frac{\omega}{\left(1+0.05 \cdot \omega^{2}\right)} .
$$

Thus the prewarping correction consists in the following substitution, also used in [15]:

$$
\omega \longrightarrow 0.95 \cdot \frac{\omega}{\left(1+0.05 \cdot \omega^{2}\right)} .
$$

Even if (21) is useful in general, taking into account that the 2D filter function $F_{D i}\left(s_{1}, s_{2}\right)$ has even parity in $s_{1}$ and $s_{2}$, that is, the variables appear as $s_{1}^{2}, s_{2}^{2}$, a more efficient pre-warping can be made in this case.

We obtain the following Chebyshev-Padé approximation on the range $\omega \in[-\pi, \pi]$ :

$$
\left(\operatorname{arctg}\left(\frac{\omega}{2}\right)\right)^{2} \cong \frac{\left(0.017251+0.218196 \cdot \omega^{2}\right)}{\left(1+0.116048 \cdot \omega^{2}\right)} .
$$

Consequently, from (19) and (22), the frequency pre-warping mapping can be written, on both frequency axes:

$$
\omega_{1,2}^{2} \longrightarrow \frac{\left(0.069+0.872783 \cdot \omega_{1,2}^{2}\right)}{\left(1+0.116048 \cdot \omega_{1,2}^{2}\right)},
$$

$$
s_{1,2}^{2} \longrightarrow \frac{-\left(0.069-0.872783 \cdot s_{1,2}^{2}\right)}{\left(1-0.116048 \cdot s_{1,2}^{2}\right)} .
$$

Let us now apply the bilinear transform on both axes; that is, we substitute relations (17) into mapping (24); we get the following simple mapping, valid on both axes $\omega_{1}$ and $\omega_{2}$ :

$$
s_{1,2}^{2} \longrightarrow 6.3868 \cdot \frac{\left(z_{1,2}^{2}-2.080651 \cdot z_{1,2}+1\right)}{\left(z_{1,2}^{2}+5.465361 \cdot z_{1,2}+1\right)} \text {. }
$$

The analog-discrete mapping (25) therefore is a corrected form of the bilinear transform including pre-warping in the entire region of interest, namely, $\omega_{1,2} \in[-\pi, \pi]$. It is noteworthy that this pre-warping does not increase the order. The mapping (25) can be written on each axis in terms of frequency $\omega$ :

$$
\omega^{2} \longrightarrow 6.3868 \cdot \frac{(1.0403-\cos \omega)}{(2.7326+\cos \omega)}
$$

This mapping is plotted in Figure 3 versus $\omega^{2}$ as curve F1 in solid line and is almost linear at least on the range $\omega \epsilon$ $[0,0.7 \pi]$, as compared to the mapping (21) — curve F2, plotted in dash line. Therefore the proposed correction compensates the distortions introduced by the bilinear transform. As shown further in the design examples, using the simple frequency mapping (25) which includes pre-warping, we obtain 2D square filters with good linearity along their axes. For the sum of frequency variables, $\omega_{1}+\omega_{2}$, the mapping (26) becomes

$$
\left(\omega_{1}+\omega_{2}\right)^{2} \longrightarrow 6.3868 \cdot \frac{\left(1.0403-\cos \left(\omega_{1}+\omega_{2}\right)\right)}{\left(2.7326+\cos \left(\omega_{1}+\omega_{2}\right)\right)},
$$

and since $\cos \left(\omega_{1}+\omega_{2}\right)=0.5 \cdot\left(z_{1} z_{2}+z_{1}^{-1} z_{2}^{-1}\right)$ we obtain the mapping

$$
\left(s_{1}+s_{2}\right)^{2} \longrightarrow 6.3868 \cdot \frac{\left(z_{1}^{2} z_{2}^{2}-2.0806 \cdot z_{1} z_{2}+1\right)}{\left(z_{1}^{2} z_{2}^{2}+5.4653 \cdot z_{1} z_{2}+1\right)}
$$

The next step is to substitute the mappings (25) and (28) into the expression (15) of $S_{\varphi}$, and we obtain a rational expression of order 4 in the complex frequency variables $z_{1}$ and $z_{2}$. Thus the discrete approximation $F_{\varphi}\left(z_{1}, z_{2}\right)$ of $S_{\varphi}\left(s_{1}, s_{2}\right)$ may be expressed in the matrix form

$$
\begin{aligned}
S_{\varphi}\left(s_{1}, s_{2}\right) \longrightarrow F_{\varphi}\left(z_{1}, z_{2}\right) & =k \cdot \frac{M_{\varphi}\left(z_{1}, z_{2}\right)}{N\left(z_{1}, z_{2}\right)} \\
& =k \cdot \frac{\left(\mathbf{z}_{\mathbf{1}} \times \mathbf{M}_{\varphi} \times \mathbf{z}_{2}^{T}\right)}{\left(\mathbf{z}_{\mathbf{1}} \times \mathbf{N} \times \mathbf{z}_{2}^{T}\right)},
\end{aligned}
$$

where the variable vectors are $\mathbf{z}_{1}=\left[\begin{array}{lllll}1 & z_{1} & z_{1}^{2} & z_{1}^{3} & z_{1}^{4}\end{array}\right]$, $\mathbf{z}_{\mathbf{2}}=\left[\begin{array}{lllll}1 & z_{2} & z_{2}^{2} & z_{2}^{3} & z_{2}^{4}\end{array}\right], k=3.1934$, and $\times$ stands for inner product.

The numerator matrix $\mathbf{M}_{\varphi}$ depends on the orientation angle $\varphi$ according to the expression

$$
\mathbf{M}_{\varphi}=\mathbf{M}_{\mathbf{0}}+\sin 2 \varphi \cdot \mathbf{M}_{\mathbf{1}}+\cos 2 \varphi \cdot \mathbf{M}_{\mathbf{2}}
$$


where $\mathbf{M}_{0}, \mathbf{M}_{1}$, and $\mathbf{M}_{2}$ are the following matrices of size $5 \times 5$ with constant elements

$$
\begin{aligned}
\mathbf{M}_{0} & =\left[\begin{array}{ccccc}
-0.25 & -0.42307 & -0.25 & 0 & 0 \\
-0.42307 & 1.47642 & -2.73526 & -1.36630 & 0 \\
-0.25 & -2.73526 & 15.036 & -2.73526 & -0.25 \\
0 & -1.36630 & -2.73526 & 1.47642 & -0.42307 \\
0 & 0 & -0.25 & -0.42307 & -0.25
\end{array}\right], \\
\mathbf{M}_{1} & =\left[\begin{array}{ccccc}
0.125 & -0.2601 & 0.125 & 0 & 0 \\
-0.2601 & -4.9499 & 3.47347 & 1.62637 & 0 \\
0.125 & 3.47347 & -7.51803 & 3.47347 & 0.125 \\
0 & 1.62637 & 3.47347 & -4.9499 & -0.2601 \\
0 & 0 & 0.125 & -0.2601 & 0.125
\end{array}\right], \\
\mathbf{M}_{2} & =\left[\begin{array}{ccccc}
0 & -0.94322 & 0 & 0 & 0 \\
0.94322 & 0 & -4.21169 & 0 & 0 \\
0 & 4.21169 & 0 & 4.21169 & 0 \\
0 & 0 & -4.21169 & 0 & 0.94322 \\
0 & 0 & 0 & -0.94322 & 0
\end{array}\right] .
\end{aligned}
$$

The denominator matrix $\mathbf{N}$ is also a constant $5 \times 5$ matrix:

$$
\mathbf{N}=\left[\begin{array}{ccccc}
0.125 & 0.68315 & 0.125 & 0 & 0 \\
0.68315 & 4.4167 & 4.4167 & 0.68315 & 0 \\
0.125 & 4.4167 & 20.6546 & 4.4167 & 0.125 \\
0 & 0.68315 & 4.4167 & 4.4167 & 0.68315 \\
0 & 0 & 0.125 & 0.68315 & 0.125
\end{array}\right]
$$

From (16) and (29), after some algebra, the matrices $\mathbf{B}_{i}, \mathbf{A}_{i}(i=$ $1,2, \ldots)$ corresponding to the numerator and denominator of $F_{D i}\left(s_{1}, s_{2}\right)$ result from similar relations:

$$
\begin{aligned}
& \mathbf{B}_{i}=p^{4} k^{2} \cdot \mathbf{M}_{\varphi} * \mathbf{M}_{\varphi}-b_{1 i} \cdot p^{2} k \cdot \mathbf{M}_{\varphi} * \mathbf{N}+b_{0 i} \cdot \mathbf{N} * \mathbf{N} \\
& \mathbf{A}_{i}=p^{4} k^{2} \cdot \mathbf{M}_{\varphi} * \mathbf{M}_{\varphi}-a_{1 i} \cdot p^{2} k \cdot \mathbf{M}_{\varphi} * \mathbf{N}+a_{0 i} \cdot \mathbf{N} * \mathbf{N}
\end{aligned}
$$

where $*$ denotes matrix convolution.

The 2D directional IIR filter will have a real-valued (zerophase) transfer function $H_{D}\left(z_{1}, z_{2}\right)$ in the complex variables $z_{1}=e^{j \omega_{1}}$ and $z_{2}=e^{j \omega_{2}}$, written in matrix form

$$
H_{D}\left(z_{1}, z_{2}\right)=\frac{B_{D}\left(z_{1}, z_{2}\right)}{A_{D}\left(z_{1}, z_{2}\right)}=\frac{\mathbf{z}_{1} \times \mathbf{B}_{\mathbf{D}} \times \mathbf{z}_{2}^{T}}{\mathbf{z}_{1} \times \mathbf{A}_{\mathbf{D}} \times \mathbf{z}_{2}^{T}},
$$

where the vectors $\mathbf{z}_{1}, \mathbf{z}_{2}$ are $\mathbf{z}_{1}=\left[\begin{array}{lllll}1 & z_{1} & \cdots & z_{1}^{N-1} & z_{1}^{N}\end{array}\right], \mathbf{z}_{2}=$ $\left[\begin{array}{lllll}1 & z_{2} & \cdots & z_{2}^{N-1} & z_{2}^{N}\end{array}\right]$ and $N$ is the directional filter order. If $\mathbf{B}_{1}, \mathbf{B}_{2}, \mathbf{A}_{1}$, and $\mathbf{A}_{2}$ are the matrices corresponding to the numerators and denominators of the two factors from (6), the matrices $\mathbf{B}_{\mathbf{D}}$ and $\mathbf{A}_{\mathbf{D}}$ of the directional filter result as convolution:

$$
\mathbf{B}_{\mathbf{D}}=\mathbf{B}_{1} * \mathbf{B}_{2}, \quad \mathbf{A}_{\mathbf{D}}=\mathbf{A}_{1} * \mathbf{A}_{2} .
$$

Finally, the square-shaped filter transfer function is given by an expression similar to (34):

$$
H_{S}\left(z_{1}, z_{2}\right)=\frac{B_{S}\left(z_{1}, z_{2}\right)}{A_{S}\left(z_{1}, z_{2}\right)}=\frac{\mathbf{z}_{1} \times \mathbf{B}_{\mathbf{S}} \times \mathbf{z}_{2}^{T}}{\mathbf{z}_{1} \times \mathbf{A}_{\mathbf{S}} \times \mathbf{z}_{2}^{T}},
$$

where the matrices $\mathbf{B}_{\mathbf{S}}$ and $\mathbf{A}_{\mathbf{S}}$ result from

$$
\mathbf{B}_{\mathbf{S}}=\mathbf{B}_{\mathbf{D}} * \mathbf{B}_{\mathbf{D}}^{R}, \quad \mathbf{A}_{\mathbf{S}}=\mathbf{A}_{\mathbf{D}} * \mathbf{A}_{\mathbf{D}}^{R},
$$

where the superscript $R$ means rotation by 90 degrees; therefore $\mathbf{A}_{\mathbf{D}}^{R}$ is the clockwise rotated version of $\mathbf{A}_{\mathbf{D}}$. This is due to the fact that the matrix pairs $\left(\mathbf{A}_{\mathrm{D}}, \mathbf{B}_{\mathrm{D}}\right)$ and $\left(\mathrm{A}_{\mathrm{D}}^{R}, \mathbf{B}_{\mathrm{D}}^{R}\right)$ correspond to two directional filters which form a right angle in the frequency plane.

To summarize, the design steps for the adjustable squareshaped filter are the following.

(a) Once adopted an 1D low-pass filter prototype of the form given by (4), its 4 th order factors are determined; therefore the coefficients $a_{0 i}, a_{1 i}, b_{0 i}$, and $b_{1 i}$ are found; if a second-order factor occurs, $c_{0 i}$ and $d_{0 i}$ are also found.

(b) For a specified orientation angle $\varphi$, the matrix $\mathbf{M}_{\varphi}$ is determined using expression (30) and the partial constant matrices (31).

(c) For a desired filter bandwidth $\omega_{0}$, specified by the parameter $p=1 / \omega_{0}$, the partial matrices $\mathbf{B}_{i}$ and $\mathbf{A}_{i}$ for each of the 4-order factors result from relations (33).

(d) The matrices $\mathbf{B}_{\mathbf{D}}$ and $\mathbf{A}_{\mathbf{D}}$ of the directional filter result as convolution of the matrices determined at the previous step, according to (35).

(e) The matrices $\mathbf{B}_{\boldsymbol{S}}$ and $\mathbf{A}_{\boldsymbol{S}}$ of the square-shaped filter result as convolution of the matrices determined at the previous step, according to (37).

\section{Design Examples}

Following the previous steps, the design of a $2 \mathrm{D}$ squareshaped filter using this method is straightforward. The bandwidth $\omega_{0}$ is specified by the parameter $p=1 / \omega_{0}$. As shown before, the $5 \times 5$ matrices $\mathbf{M}_{0}, \mathbf{M}_{1}, \mathbf{M}_{2}$, and $\mathbf{N}$ are constant once specified the $1 \mathrm{D}$ prototype $H_{P}(\omega)$, in our case given by (6).

The first design example is the diamond-type filter whose frequency response $H_{S 1}\left(\omega_{1}, \omega_{2}\right)$ is shown in Figure 4(a). In order to obtain a diamond filter as in Figure 1(a), the orientation angle must be $\operatorname{set} \varphi=\pi / 4$. Ideally, the bandwidth (cut-off frequency) should be $\omega_{0}=\pi / \sqrt{2}$, corresponding to $p=0.45$. However, due to the inherent errors and the finite slope of the filter transition region, using these values would lead to a filter with large distortions towards the margins of the frequency plane. Using instead the value $p=0.6$, corresponding to a bandwidth $\omega_{0}=0.53 \pi$, the filter will have a slightly narrower bandwidth but also smaller distortions. Also the contour plot shows a satisfactory linearity on the four sides of the square-shaped section. In Figures 4(c) and 4(d), the frequency responses and contour plots are displayed for the two directional component filters $H_{D 1}\left(z_{1}, z_{2}\right), H_{D 2}\left(z_{1}, z_{2}\right)$. The square-shaped filter characteristic results as a product of the two directional characteristics.

In Figures 5 and 6, the frequency responses and contour plots are shown, for other square-shaped filters with different 


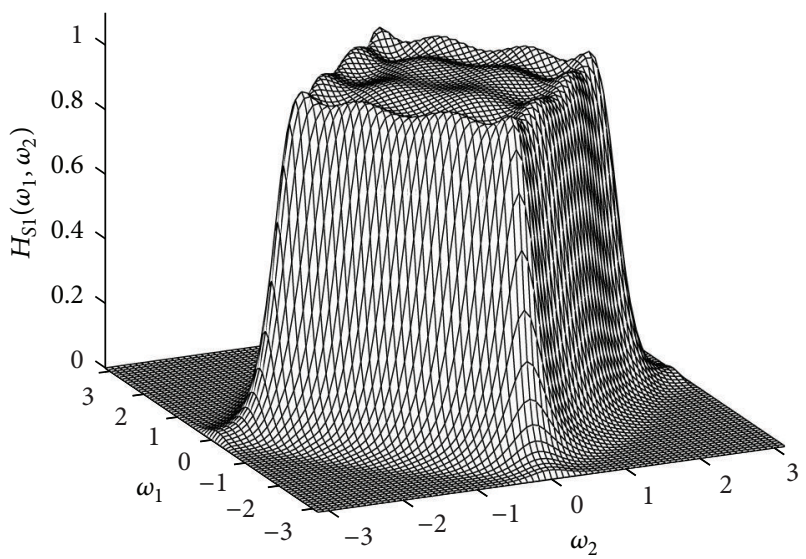

(a)

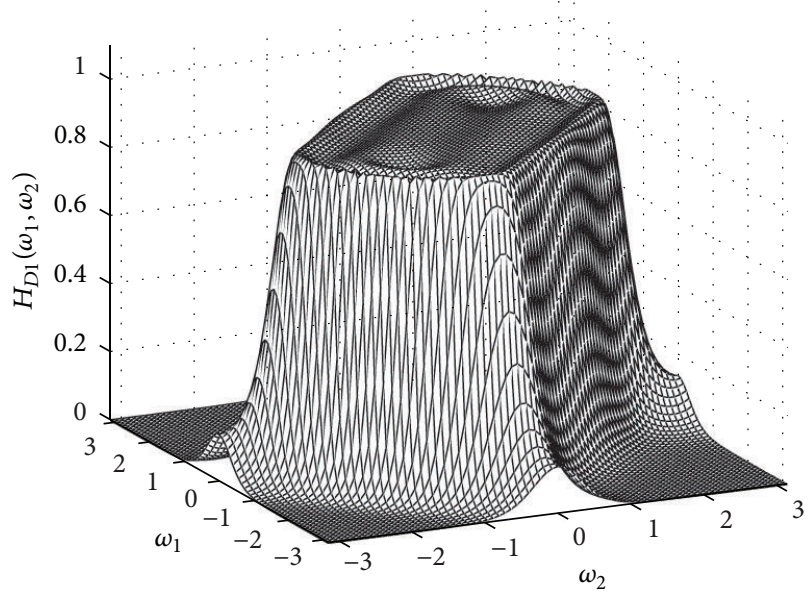

(c)

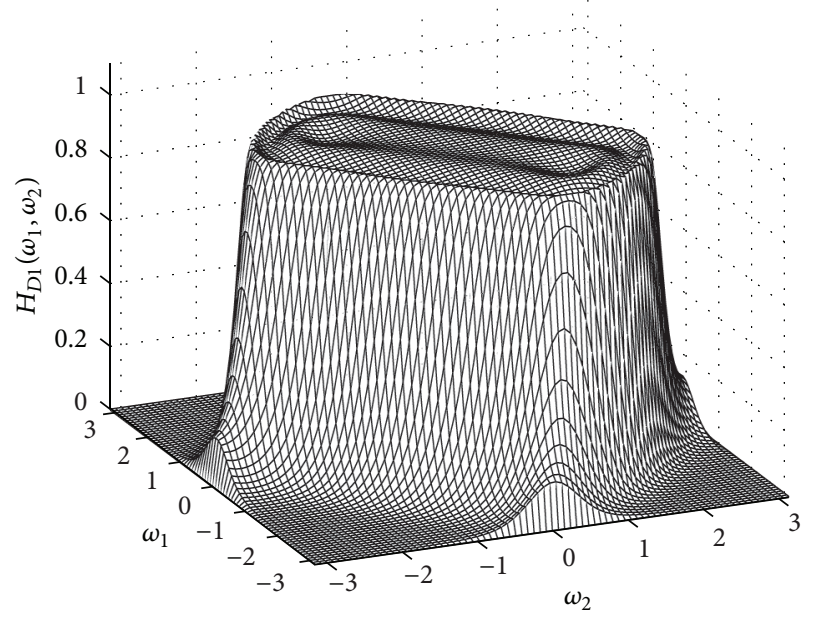

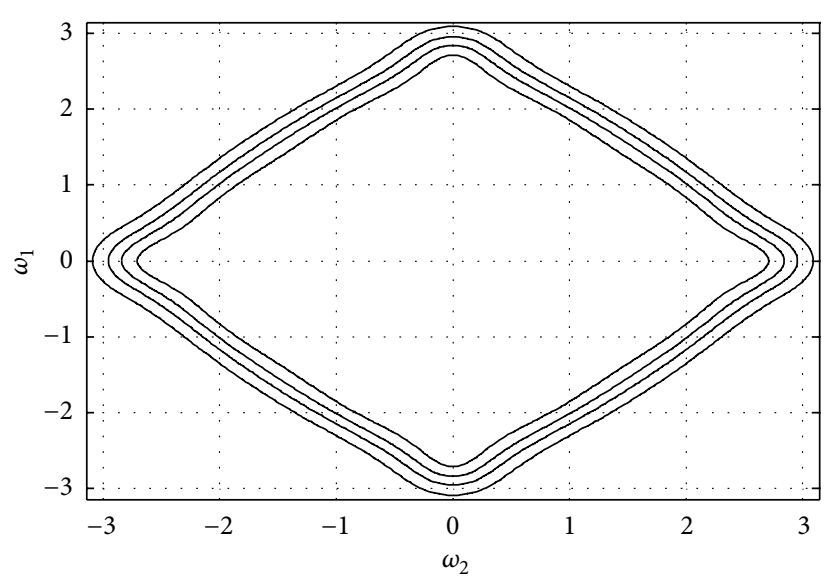

(b)
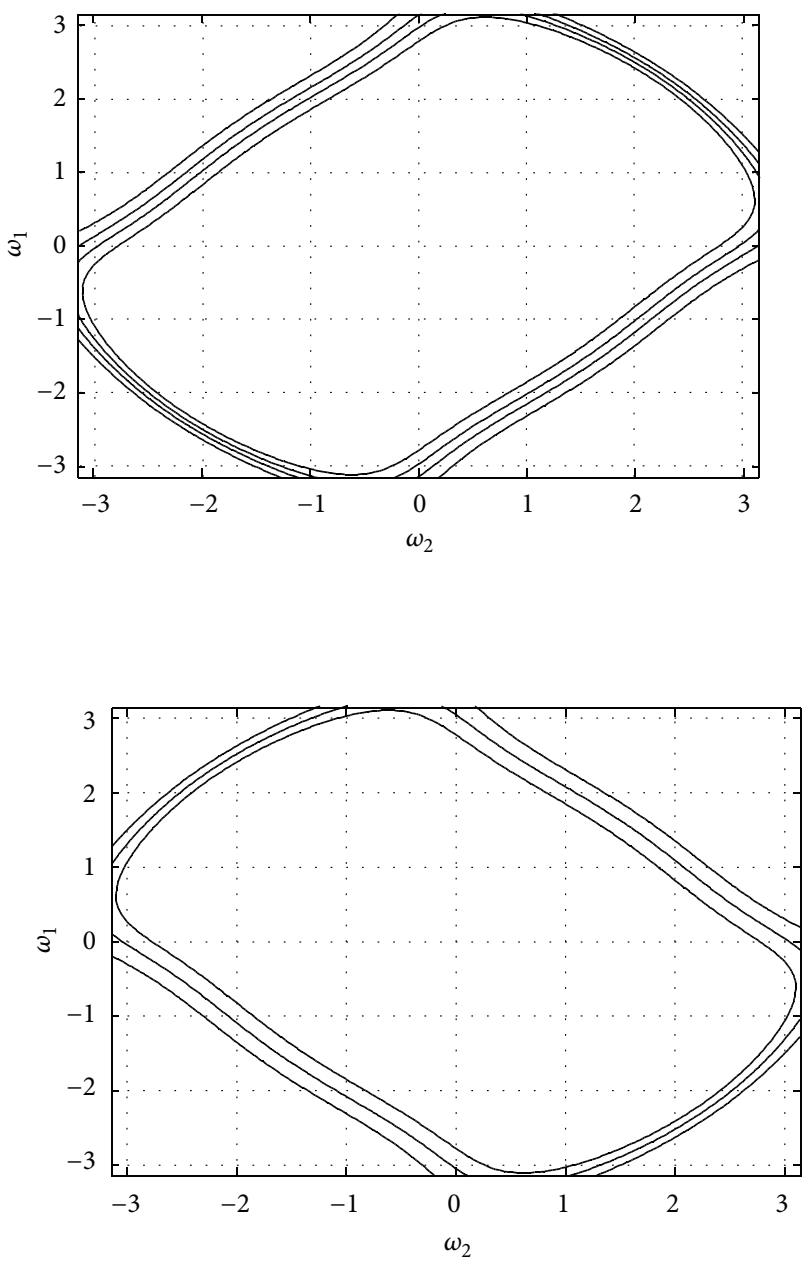

(d)

FIGURE 4: (a) Frequency response of a square-shaped filter with orientation $\varphi=\pi / 4$ and $p=0.6$ (bandwidth $\omega_{0}=1.66 \cong 0.53 \pi$ ); (b) contour plot of this filter; (c), (d) frequency responses and contour plots of the two partial oriented filters. 


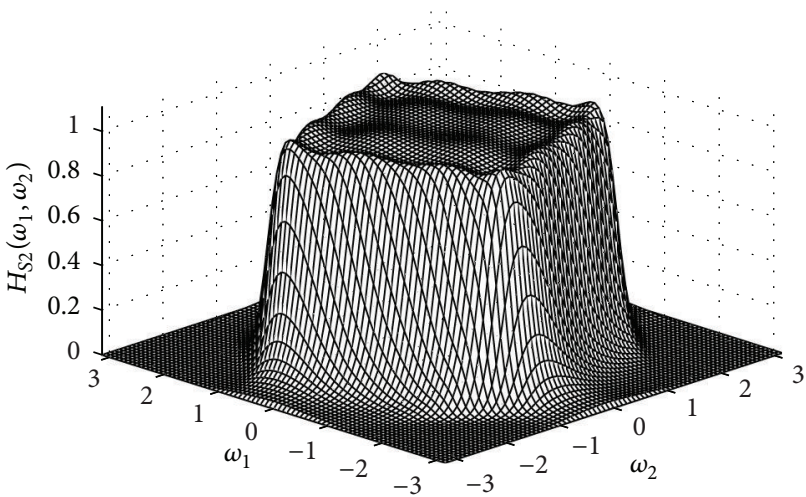

(a)

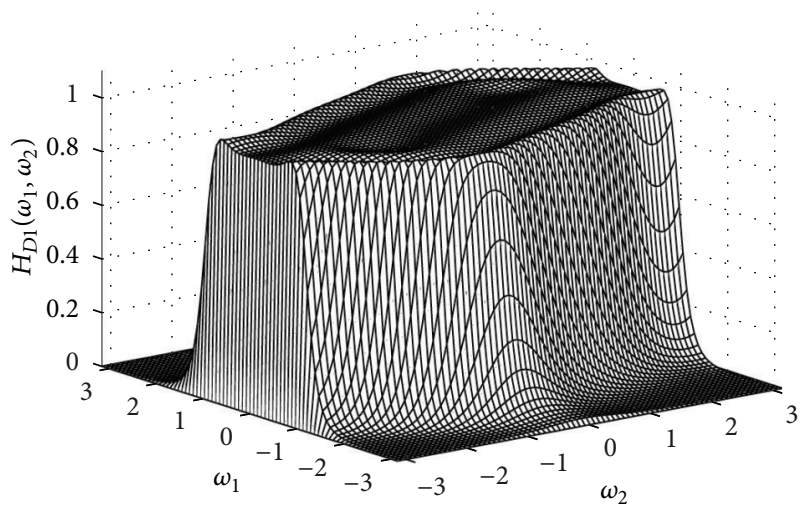

(c)

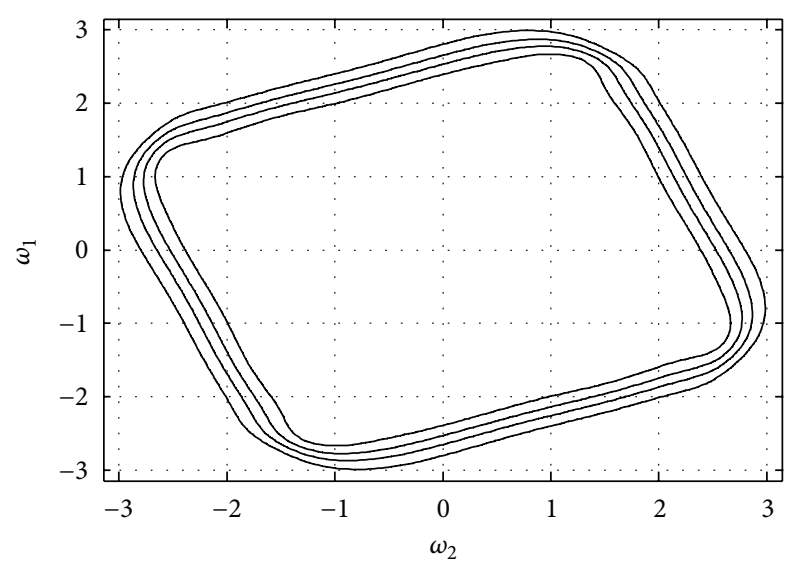

(b)

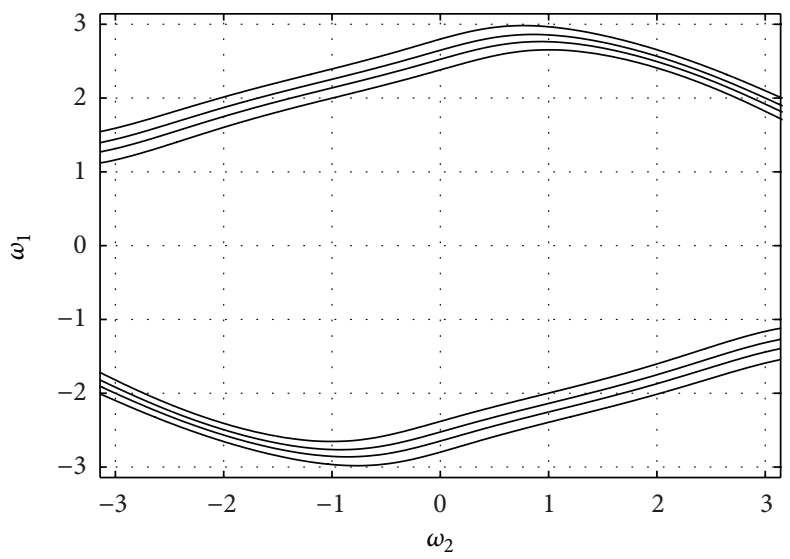

(d)

FIGURE 5: (a) Frequency response of a square-shaped filter with orientation $\varphi=3 \pi / 8$ and $p=0.54$ (bandwidth $\left.\omega_{0}=1.85 \cong 0.589 \pi\right)$; $($ b) contour plot of this filter; (c), (d) frequency response and contour plot of one oriented filter.

specifications, that is, bandwidth and orientation. It can be noticed that the filter characteristics have satisfactory steepness and relatively low ripple distortions in the passband region. Of course, better characteristics can be obtained using a higher order $1 \mathrm{D}$ prototype filter, that is, a more accurate approximation of the Butterworth LP filter. This however would increase the order of the $2 \mathrm{D}$ square-shaped filter.

A more general filter of this kind can also be derived, namely, a rhomboidal-shaped filter, in which the two component filters are not in a right angle to each other. Furthermore, a directional filter like the ones in Figure 4(c) or Figure 6(c) can be used independently, especially if it has a narrow bandwidth and can be used to extract lines with different spatial orientations from an image by tuning it to specified directions.

Even if this analytical design method may not yield optimal filters in terms of complexity, as is generally the case with techniques based on numerical optimization, the advantage of having parametric, closed form expressions for the filter matrices is the possibility of tuning or adjusting the 2D filter for a new set of specifications-in our case bandwidth and orientation-without the need to resume the design process every time.

Stability of the proposed filters is also an important issue. The stability problem for $2 \mathrm{D}$ filters is much more difficult than for $1 \mathrm{D}$ filters. There exist various stability criteria $[7,8]$ and also stabilization methods which can be applied for some unstable filters $[9,10]$. A full stability analysis of the proposed adjustable 2D filters will be approached in further work.

The main goal of the paper was to present in detail this analytical design method and some relevant design examples for these filters. Since they can select adjustable square or rhomboidal regions in the frequency plane, they may have interesting applications in image processing. Their applications in image filtering will be investigated in future work on this topic.

\section{Conclusions}

An analytical design method for recursive zero-phase squareshaped filters was proposed. The design is based on analog 


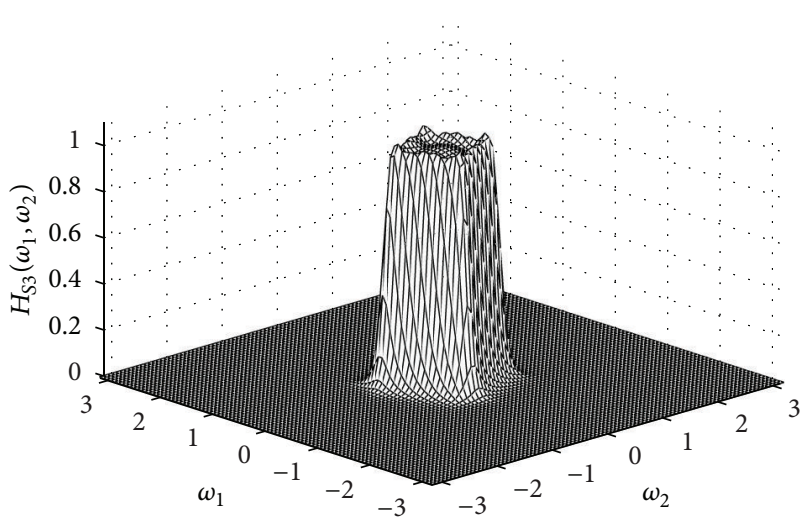

(a)

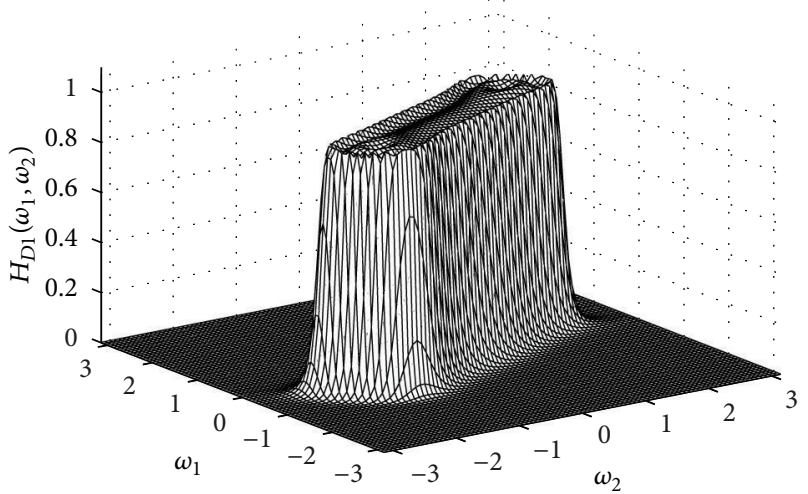

(c)

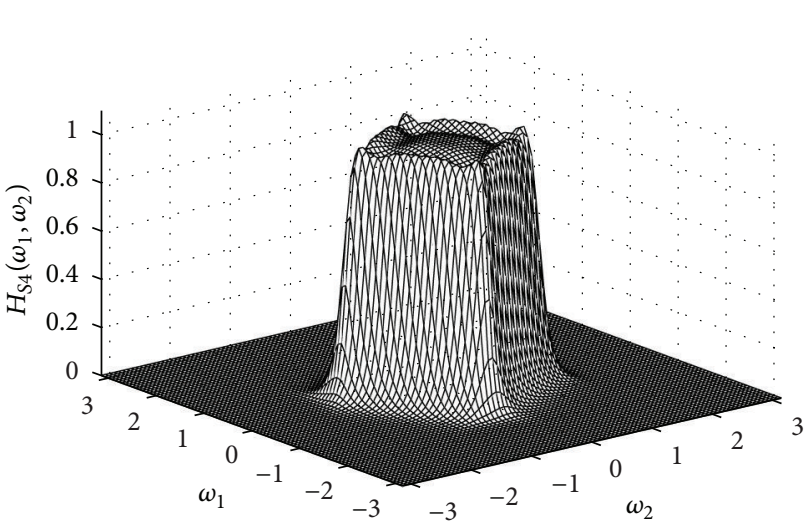

(e)

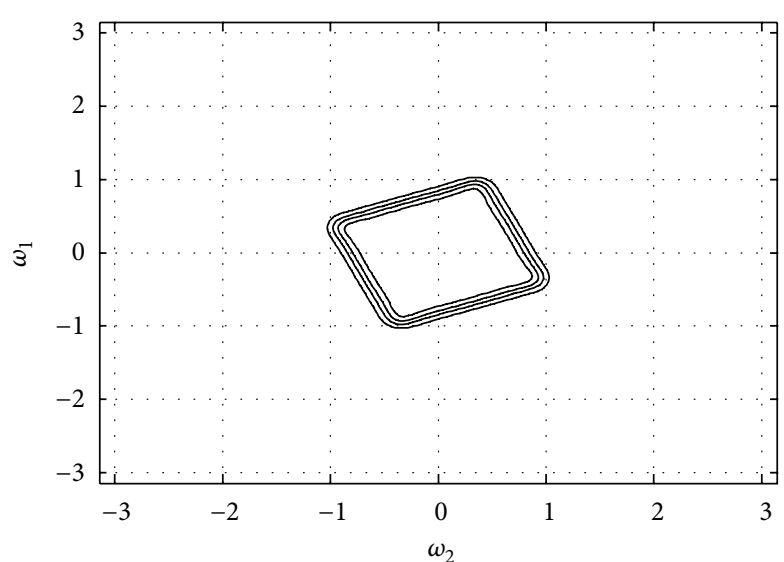

(b)

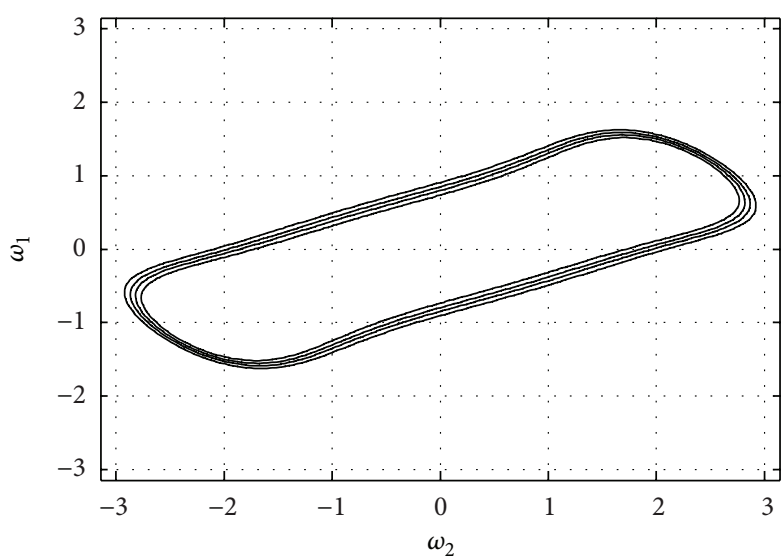

(d)

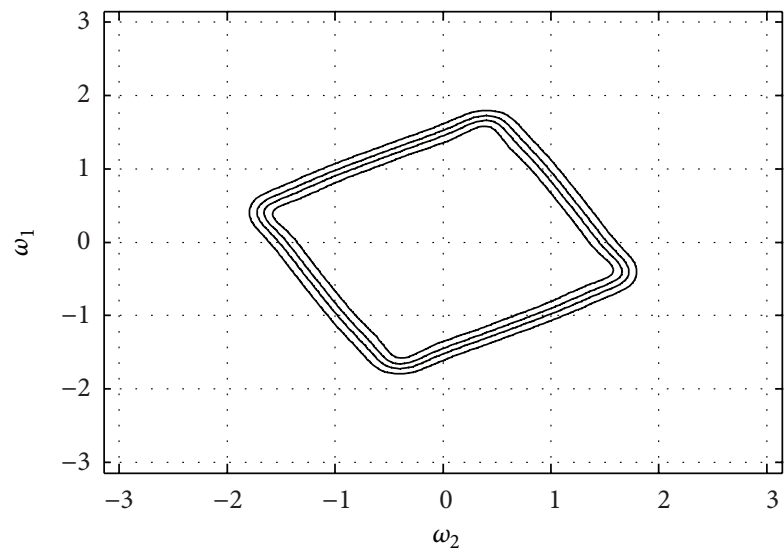

(f)

FIGURE 6: (a) Frequency response of a square-shaped filter with orientation $\varphi=3 \pi / 8$ and $p=1.6$ (bandwidth $\left.\omega_{0}=0.625 \cong 0.2 \pi\right)$; (b) contour plot of this filter; (c), (d) frequency response and contour plot of one oriented filter; (e), (f) frequency response and contour plot for a square-shaped filter with $\varphi=\pi / 3$ and $p=1$ (bandwidth $\omega_{0}=1 \cong 0.318 \pi$ ).

zero-phase low-pass prototypes with specified parameters. As 1D prototype, a Butterworth filter was used, and, using an efficient rational approximation, a zero-phase filter small passband ripple was derived. The resulted $2 \mathrm{D}$ filter is parametric or adjustable in the sense that the specified parametersbandwidth and orientation angle-appear explicitly in the final filter matrices. The method includes the bilinear transform applied along the two axes and uses a frequency pre-warping to compensate for distortions. This leads to a particular frequency mapping, which is applied to the $1 \mathrm{D}$ prototype in order to obtain the $2 \mathrm{D}$ filter. This design technique is relatively simple, efficient and versatile, in the 
sense that, by changing the specifications, the new 2D filter matrices result directly, without the need to resume the entire design process all the way from the start.

\section{References}

[1] W. S. Lu and A. Antoniou, Two-Dimensional Digital Filters, CRC Press, 1992.

[2] S. Chakrabarti and S. K. Mitra, "Design of two-dimensional digital filters via spectral transformations," Proceedings of the IEEE, vol. 65, no. 6, pp. 905-914, 1977.

[3] K. Hirano and J. K. Aggarwal, "Design of two-dimensional recursive digital filters," IEEE Transactions on Circuits and Systems, vol. 25, no. 12, pp. 1066-1076, 1978.

[4] B. Dumitrescu, "Optimization of two-dimensional IIR filters with nonseparable and separable denominator," IEEE Transactions on Signal Processing, vol. 53, no. 5, pp. 1768-1777, 2005.

[5] D. T. Nguyen and M. N. S. Swamy, "A class of 2-D separable denominator filters designed via the McClellan transform," IEEE transactions on circuits and systems, vol. 33, no. 9, pp. 874$881,1986$.

[6] L. Harn and B. A. Shenoi, "Design of stable two-dimensional IIR filters using digital spectral transformations," IEEE transactions on circuits and systems, vol. 33, no. 5, pp. 483-490, 1986.

[7] X. Nie, R. Unbehauen, and B. Anhaupl, "On the design and realization of a class of tunable 2-D digital filters," in Proceedings of the IEEE International Symposium on Circuits and Systems (ISCAS '91), pp. 452-455, Singapore, June 1991.

[8] N. E. Mastorakis, "New necessary stability conditions for 2-D systems," IEEE Transactions on Circuits and Systems I, vol. 47, no. 7, pp. 1103-1105, 2000.

[9] T. Miyata, N. Aikawa, Y. Sugita, and T. Yoshikawa, "A design method for separable-denominator 2D IIR filters using a stability criterion based on the system matrix," in Proceedings of the 15th IEEE International Conference on Electronics, Circuits and Systems (ICECS '08), pp. 826-829, Msida, Malta, September 2008.

[10] E. I. Jury, V. R. Kolavennu, and B. D. O. Anderson, "Stabilization of certain two-dimensional recursive digital filters," Proceedings of the IEEE, vol. 65, no. 6, pp. 887-892, 1977.

[11] N. Damera-Venkata, M. Venkataraman, M. S. Hrishikesh, and P. S. Reddy, "STabilization of 2-D Recursive Digital Filters by the DHT method," IEEE Transactions on Circuits and Systems II, vol. 46, no. 1, pp. 85-88, 1999.

[12] D. V. Tosic, A. Mojsilovic, and M. Popovic, "Symbolic approach to 2D biorthogonal diamond-shaped filter design," in Proceedings of the 21st International Conference on Microelectronics (MIEL '97), vol. 2, pp. 709-712, Niš, Yugoslavia, September 1997.

[13] S.-H. Low and Y.-C. Lim, "A new approach to design sharp diamond-shaped filters," Signal Processing, vol. 67, no. 1, pp. 3548, 1998.

[14] Y. C. Lim and S. H. Low, "Synthesis of sharp diamond-shaped filters using the frequency response masking approach," in Proceedings of the IEEE International Conference on Acoustics, Speech, and Signal Processing (ICASSP '97), pp. 2181-2184, April 1997.

[15] R. Matei, "A new design method for IIR diamond-shaped filters," in Proceedings of the 18th European Signal Processing Conference (EUSIPCO '10), pp. 65-69, Aalborg, Denmark, August 2010. 

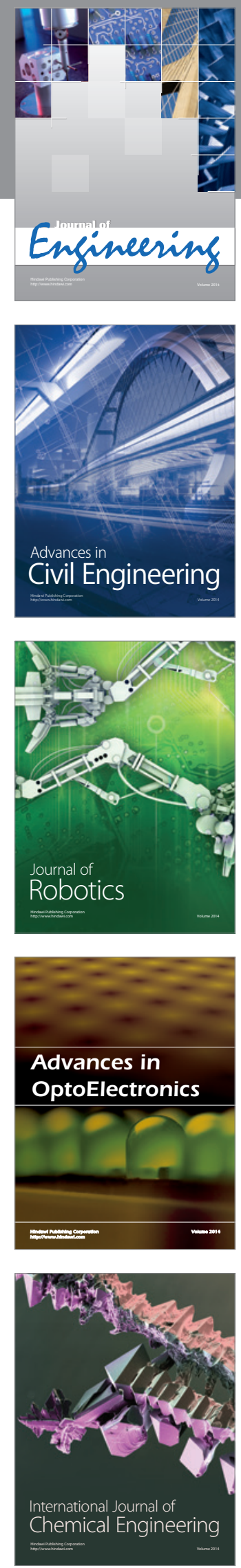

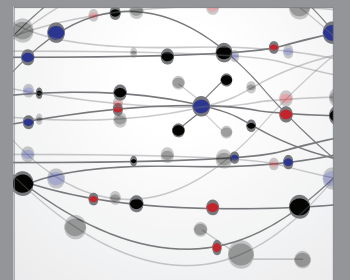

The Scientific World Journal
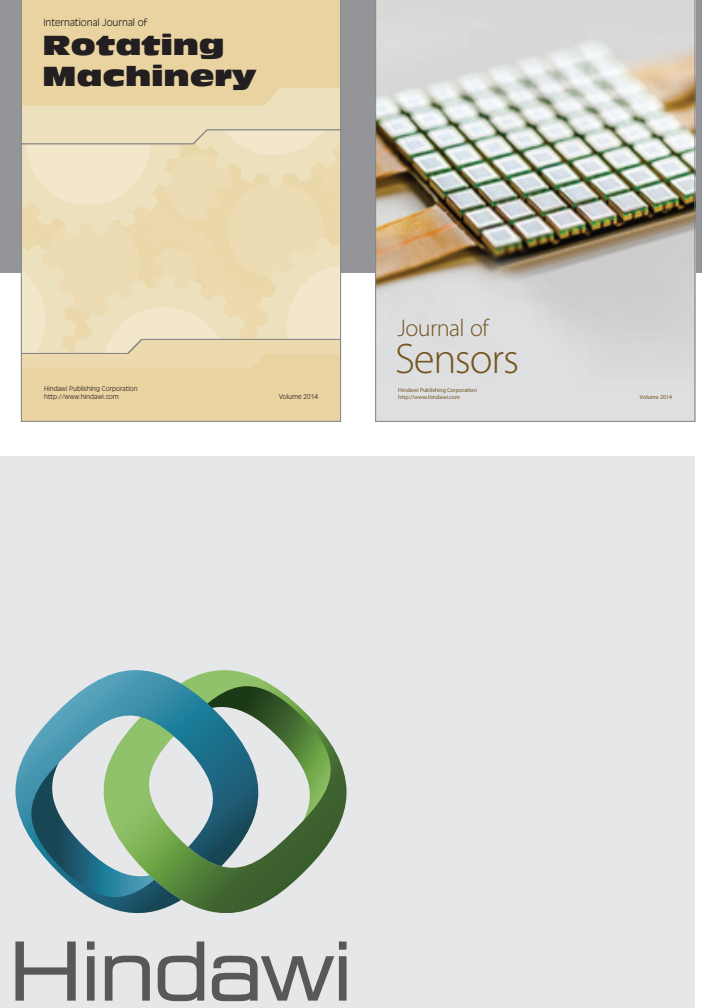

Submit your manuscripts at http://www.hindawi.com
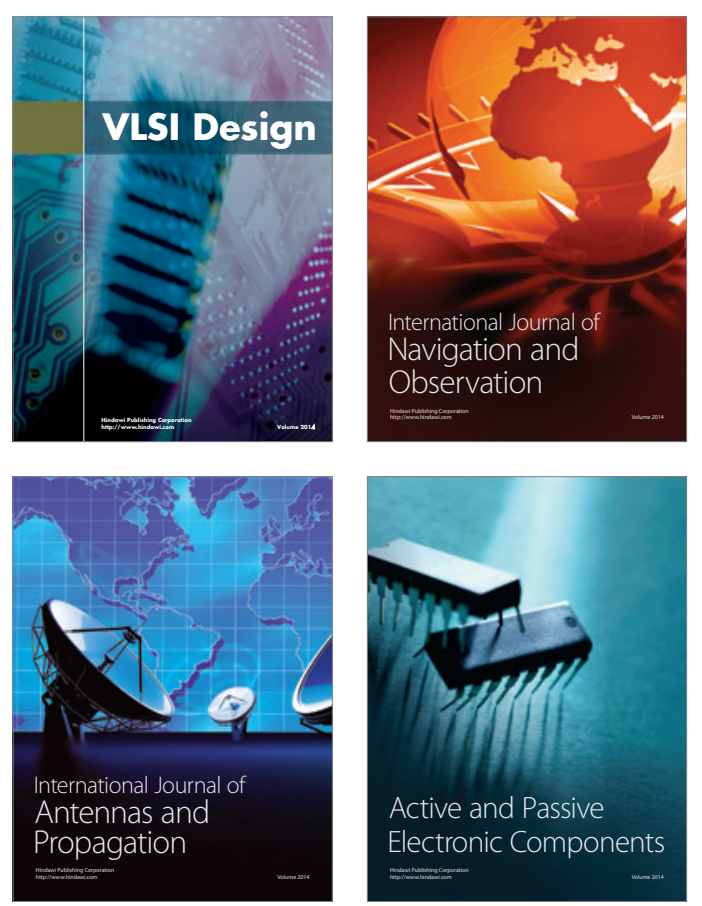
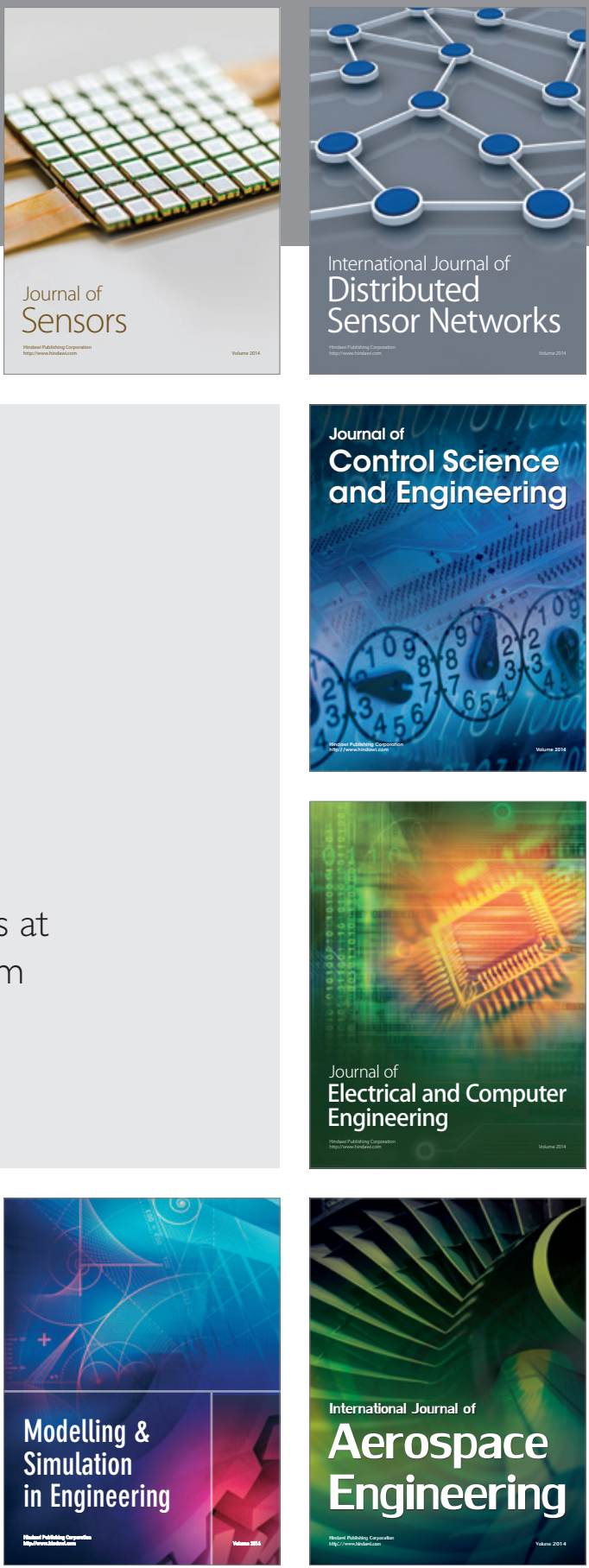

Journal of

Control Science

and Engineering
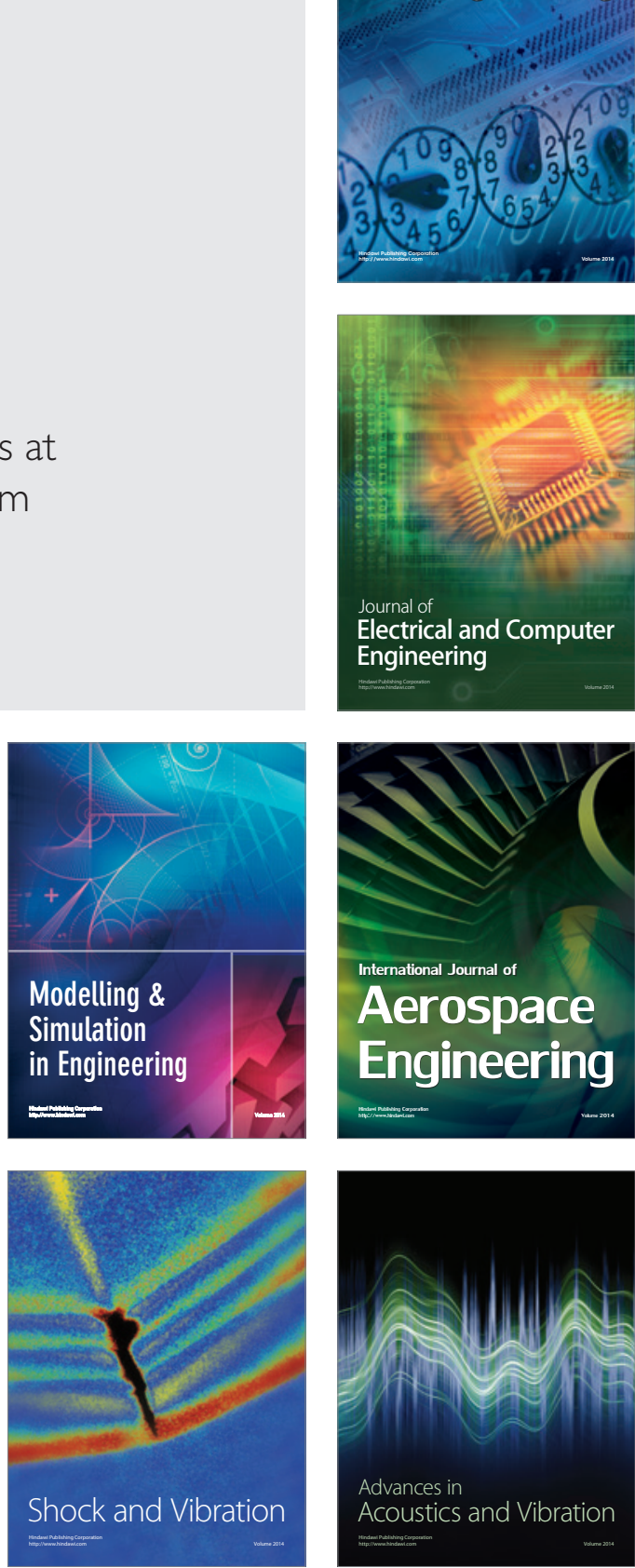